\title{
Reaching Consensus on Rumors
}

\author{
U. Merlone $e^{1, a}$, D. Radi ${ }^{1, b, *}$ \\ ${ }^{a}$ Department of of Psychology, University of Torino, Italy \\ ${ }^{b}$ Department of Management, Economics and Quantitative Methods, University of Bergamo, Italy
}

\begin{abstract}
An important contribution in sociophysics is the Galam's model of rumors spreading. This model provides an explanation of rumors spreading in a population and explains some interesting social phenomena such as the diffusion of hoaxes. In this paper the model has been reformulated as a Markov process highlighting the stochastic nature of the phenomena. This formalization allows us to derive conditions for consensus to be reached and for the existence of some interesting phenomena such as the emergence of impasses. The proposed formulation allows a deeper and more comprehensive analysis of the diffusion of rumors.
\end{abstract}

Keywords: Sociophysics, Binary games, Hypergeometric random variable, Stochastic matrix, Absorbing Markov Chain.

\section{Introduction}

Consensus in groups has attracted interest from different disciplines, namely social psychology, economics, sociology and political sciences. For example, the influence of social pressure exercised by a minority is studied in [17] where the Authors analyze how behavioral style may be a general source of influence. The process by which a group reaches consensus has been formalized and mathematically analyzed in [9] and [3] providing also simple conditions determining whether it is possible for the group to reach consensus. Another important contribution is provided by [16] which introduces a time-changing influential matrix and provides a sufficient condition to obtain convergence on the influential weights. More recent contributions based on the same modeling framework account for strategic interaction and study the existence of possible consensus equilibria when strategic interaction and social influence are combined together. For example, [7] incorporates in a DeGroot-like model, see again [9], the possibility that agents misrepresent their opinion with the intent of reaching a conformity. Indeed, adopting a behavior that is different from the others might cause disutility. In this case, the combination of this strategic acting and the social influence requires additional conditions to reach consensus. Following a similar idea, [8] introduces an extension of the classical DeGroot model of

\footnotetext{
${ }^{*}$ Corresponding author

Email addresses: ugo.merlone@unito.it (U. Merlone), davide.radi@unibg.it (D. Radi)
} 
opinion formation for studying the transmission of cultural traits in an overlapping generation setting, where parents strategically display a cultural trait to influence their children.

Sociophysics as well, has devoted a lot of attention to social influence, starting from the pioneering works by Galam (see, e.g., [14]) to recent contributions, such as [18]; for a review see [13] and [20]. In his contributions [11] and [12], Galam moves the perspective from within the group to a whole population whose individuals try to choose an opinion (true or false) on a rumor on the basis of repeated discussions in social gatherings. At each of them, a small group of people get together and line up with a consensual opinion in which everyone agrees with the majority inside the group. His model provides a plausible explanation for the diffusion or self-propagation of rumors through free public debates, such as the temporary diffusion of the so called Pentagon French hoax, according to which no plane crashed on the Pentagon on September the $11^{\text {th }}$. Indeed, although initially supported only by a minority of the population, this rumor started to propagate with an astonishing and unexpected adhesion till the moment in which a strong media campaign carried out by newspapers reversed the process.

In the Galam's formulation of the model the binomial distribution has been used to approximate the probability of having a specific table seating configuration. The use of the binomial distribution is a good modeling approximation as long as populations with a large number of agents and small size discussion group are considered. Nevertheless, this approach may create distortions in the prediction of the dynamics of rumors in small communities. In order to deal with this problem, we formalize the process as an absorbing Markov chain, in which the states of the process correspond to the number of agents holding one opinion. Our approach extends Galam's analysis providing further results and precision. The goal is to have further information about the process of diffusion of opinions which are not possible to obtain using the original Galam's formulation. In particular, we aim to investigate thoroughly the evolution of the rumors spreading process identifying all the absorbing states and computing the probability to reach each of them.

The structure of the paper is the following: In Section 2 the model is described as an absorbing Markov chain. In Section 3 the stochastic killing point is defined. This is the stochastic version of the killing point introduced in [12]. In Section 4 the formula for the conditional expected values is provided. Finally, in Section 5 further possible developments of the model are suggested.

\section{Formalization of the process}

Consider a $N$ person finite population and assume that only two opinions, ' + ' and '-', are possible. Assume that at time $t=0,1, \ldots$ each individual holds either one or the other opinion and $Y^{t}$ denotes the number of those holding opinion ' + ' at time $t$. The set of possible states of the population with respect to opinion ' + ' is therefore $S=\{0,1, \ldots, N\}$, where state 0 means consensus has been reached on opinion '-' 
while, on the contrary, state $N$ means consensus has been reached on opinion '+'. Not all the states in $S$ are necessarily feasible. State feasibility depends on aspects, which will be introduced later, such as social space and discussion functions .

As in [12], [5] and [6] the interaction takes place at different size tables. The social space, where the discussion takes place, is the set of tables $\mathcal{N}=\left\{T_{1}, T_{2}, \ldots, T_{L}\right\}$ with ${ }^{1} L<N$. Let $\left|T_{r}\right|$ be the size of table $T_{r}$ with $\sum_{T_{r} \in \mathcal{N}}\left|T_{r}\right|=N$. The table sizes, i.e., the number of people that can be seated at a given table, can be summarized in vector $\mathbf{n}=\left(\left|T_{1}\right|,\left|T_{2}\right|, \ldots,\left|T_{L}\right|\right) \in \mathbb{R}^{L}$. As the number of seats at each table is the only relevant variable, the social space can be denoted either by $\mathcal{N}$ or $\mathbf{n}$; furthermore we assume as in [12] that the social space remains the same during the whole process.

Given a social space $\mathcal{N}$, we can determine how may tables have size $k$ as follows

$$
\tau_{k}=\sum_{T_{r} \in \mathcal{N}} \delta_{k,\left|T_{r}\right|}
$$

where $\delta_{k,\left|T_{r}\right|}$ is the Kronecker's delta.

As we assume the social space being fixed over time, the probability $a_{k}$ to be seated at a size $k$ table is stationary and can be determined as follows

$$
a_{k}=\frac{k}{N} \tau_{k} \text { with } k=1, \ldots, K,
$$

where $K$ is the number of seats of the largest size table in the social space. Given the social space $\mathcal{N}=$ $\left\{T_{1}, T_{2}, \ldots, T_{L}\right\}$, vector $\mathbf{y}=\left(y_{1}, y_{2}, \ldots, y_{L}\right)$ indicates a generic seating configuration where $y_{1}$ agents with opinion ' + ' are seated at table $T_{1}, y_{2}$ are seated at table $T_{2}, \ldots$ and $y_{L}$ are seated at table $T_{L}$, with the obvious feasibility conditions $0 \leq y_{r} \leq\left|T_{r}\right|, r=1,2, \ldots, L$. Therefore entries in $\mathbf{y}$ depend $^{2}$ on the social space.

Assuming $y \in S$ agents with opinion ' + ', let us introduce the set $\Omega_{y}$ as

$$
\Omega_{y}=\left\{\mathbf{y}: 0 \leq y_{r} \leq \min \left(\left|T_{r}\right|, y\right), r=1,2, \ldots, L \text { and } \sum_{r=1}^{L} y_{r}=y\right\} .
$$

This set consists of all possible seating configurations of $y$ agents with opinion ' + ' given the social space.

The probability of each seating configuration $\mathbf{y} \in \Omega_{y}, \forall y \in S$ can be computed as follows

Proposition 1. Assuming all seating configurations are equiprobable, then the probability of configuration

\footnotetext{
${ }^{1}$ The case $L=N$, i.e. all table of dimension 1, is trivial and therefore will not be considered.

${ }^{2}$ For the sake of simplicity we can avoid using the heavier notation $\mathbf{y}_{\mathbf{n}}$ as the social space is assume fixed.
} 
$\mathbf{y} \in \Omega_{y}, \forall y \in S$ is

$$
P_{y}(\mathbf{y})=\frac{\left(\begin{array}{c}
y \\
y_{1}, y_{2}, \ldots, y_{L}
\end{array}\right)\left(\begin{array}{c}
N-y \\
\left|T_{1}\right|-y_{1},\left|T_{2}\right|-y_{2}, \ldots,\left|T_{L}\right|-y_{L}
\end{array}\right)}{N}=\frac{\left(\begin{array}{c}
\left|T_{1}\right| \\
y_{1}
\end{array}\right) \cdots\left(\begin{array}{c}
\left|T_{L}\right| \\
y_{L}
\end{array}\right)}{\left(\begin{array}{c}
N \\
y
\end{array}\right)}
$$

where $\left(\begin{array}{c}b \\ b_{1}, b_{2}, \ldots, b_{L}\end{array}\right)$ is a multinomial coefficient. $P_{y}(\mathbf{y}): \Omega_{y} \rightarrow[0,1]$ is a probability mass function.

Proof. Given a $N$-agents population and a social space $\mathbf{n}=\left(\left|T_{1}\right|,\left|T_{2}\right|, \ldots,\left|T_{L}\right|\right)$. The number of all the possible combinations of the $N$ agents at the $L$ tables of social space is $\left(\begin{array}{c}N \\ \left|T_{1}\right|,\left|T_{2}\right|, \ldots,\left|T_{L}\right|\end{array}\right)$ and the number of possible combinations of $y$ agents with opinion ' + ' and $N-y$ agents with opinion '-' for which we have the seating configuration $\mathbf{y}=\left(y_{1}, y_{2}, \ldots, y_{L}\right)$ is

$$
\left(\begin{array}{c}
y \\
y_{1}, y_{2}, \ldots, y_{L}
\end{array}\right)\left(\begin{array}{c}
N-y \\
\left|T_{1}\right|-y_{1},\left|T_{2}\right|-y_{2}, \ldots,\left|T_{L}\right|-y_{L}
\end{array}\right)
$$

Since all the $\left(\begin{array}{c}N \\ \left|T_{1}\right|,\left|T_{2}\right|, \ldots,\left|T_{L}\right|\end{array}\right)$ possible combinations are equiprobable, the probability of the seating configuration $\mathbf{y}$ is given by

$$
P_{y}(\mathbf{y})=\frac{\left(\begin{array}{c}
y \\
y_{1}, y_{2}, \ldots, y_{L}
\end{array}\right)\left(\begin{array}{c}
N-y \\
\left|T_{1}\right|-y_{1},\left|T_{2}\right|-y_{2}, \ldots,\left|T_{L}\right|-y_{L}
\end{array}\right)}{N} .
$$

By multinomial coefficients properties and Chu-Vandermonde's identity, it is clear that $P_{y}(\mathbf{y}): \Omega_{y} \rightarrow[0,1]$ is a probability mass function.

As in [12] the dynamics of the system evolves at discrete times, $t \in \mathbb{N}$. At each iteration, agents are randomly seated at the tables. At the different tables, individuals line up with a consensual opinion in which everyone agrees with the majority inside the group and afterwards they leave the tables. As in [12], when an opinion is exactly split at an even size table the outcome is determined assuming a bias in favor of opinion '-'. A single step of the opinion dynamics is illustrated in the example provided in Figure 1.

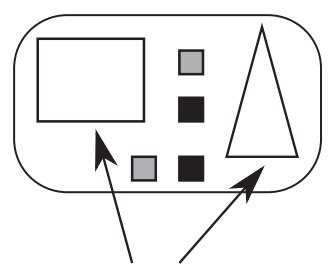

Empty social spaces

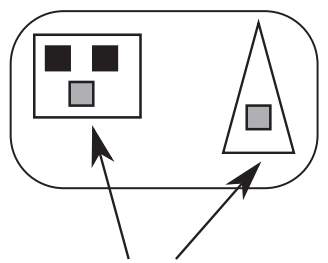

Social gathering before discussion

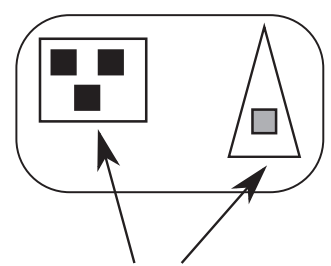

Social gathering after discussion

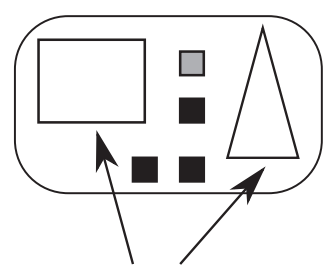

Empty social spaces

Figure 1: A one step opinion dynamics. First stage, people sharing the two opinions are moving around. Grey have opinion ' + ' while black have opinion '-'. No discussion is occurring with 2 gray and 2 black. Second stage right, people are partitioned in groups of various sizes from one to three and no change of opinion occurs. Third stage, within each group consensus has been reached. As a result, they are now 1 gray and 3 black. Last stage, people are again moving around with no discussion.

The described mechanism of diffusion of rumors can be formalized as a memoryless stochastic process $\left(Y^{t}\right)_{t \geq 0}$ where $Y^{t}$ is a random variable representing the number of agents with opinion ' + ' at time $t$. To 
this purpose we formalize the change of opinion among agents seated at table $T_{r}$ by introducing the majority rule discussion function $D$ :

$$
D\left(y_{r},\left|T_{r}\right|\right)=\left\{\begin{array}{ll}
\left|T_{r}\right| & \text { if } y_{r} \geq\left\lfloor\frac{\left|T_{r}\right|}{2}\right\rfloor+1 \\
0 & \text { if } y_{r} \leq\left\lfloor\frac{\left|T_{r}\right|}{2}\right\rfloor
\end{array},\right.
$$

where $\lfloor\cdot\rfloor$ gives the greatest integer less than or equal to the argument.

The step function $D$ describes the biased discussion rule as in [12]. Different opinion formation rules within groups can be modeled with appropriate discussion functions. For example, when the discussion is not biased it is sufficient to consider:

$$
D^{\prime}\left(y_{r},\left|T_{r}\right|\right)=\left\{\begin{array}{ll}
\left|T_{r}\right| & \text { if } y_{r}>\left|T_{r}\right| / 2 \\
n_{r} / 2 & \text { if } y_{r}=\left|T_{r}\right| / 2 \\
0 & \text { if } y_{r}<\left|T_{r}\right| / 2
\end{array} .\right.
$$

In the following, consistently to [12], we will consider only discussion function (4).

The social space and the majority rule discussion function determine the set of feasible states $F_{\mathbf{n}} \subseteq S$. According to function $D$, at each table $T_{r}$ the discussion ends with either $\left|T_{r}\right|$ or 0 agents with opinion ' + '. It follows that state $s$ is feasible if and only if there exists at least a table combination $C_{s} \subseteq \mathcal{N}$ such that $s=\sum_{T_{r} \in C_{s}}\left|T_{r}\right|$. Generally, there are more than one table combination giving state $s$.

The next step to formalize the stochastic process is to define the transition probability from one state to another in the state space $F_{\mathbf{n}}$ under majority rule discussion functions. Consider the set $\omega_{i, j} \subseteq \Omega_{i}$ of seating configurations such that, starting with $i$ agents with opinion ' + ', after the discussion $j$ agents end up with opinion ' + ' (under majority rule discussion function); formally

$$
\omega_{i, j}=\left\{\mathbf{y}: \mathbf{y} \in \Omega_{i} \text { and } \sum_{r=1}^{L} D\left(y_{r},\left|T_{r}\right|\right)=j\right\} .
$$

Therefore, starting with $i$ agents with opinion ' + ', the probability of ending up after a discussion with $j$ agents having opinion ' + ' is

$$
p_{i, j}=\sum_{\mathbf{y} \in \omega_{i, j}} P_{i}(\mathbf{y})
$$

where $P_{i}(\mathbf{y})$ is defined as in Proposition 1. It is clear that $p_{i, j}$ is the transition probability from state $i$ to state $j$, with $i, j \in F_{\mathbf{n}}$.

From the transition probability measure $p_{i j}, \forall i, j \in F_{\mathbf{n}}$, it is possible to define the transition matrix $M_{\mathbf{n}}=\left(p_{i, j}\right)_{i, j \in F_{\mathbf{n}}}$, from which we have

Theorem 2. The stochastic process $\left(Y^{t}\right)_{t \geq 0}$ on $F_{\mathbf{n}}$ described by the pair $\left(M_{\mathbf{n}}, F_{\mathbf{n}}\right)$ is an absorbing Markov 
chain such that states 0 and $N$ are always absorbing. There exists one additional absorbing state $s=(N / 2)$ if and only if the social space is composed exclusively of two odd-size tables of the same size.

See Appendix for the proof.

This is the main result of the paper as it provides the conditions for reaching consensus. In particular, it states that the two states representing consensus either in opinion '-' or '+', i.e., 0 and $N$, are always absorbing. Furthermore, excluding the very specific case of two odd tables of equal size, it is always possible to reach consensus starting from a mixed opinion, that is, from states different from 0 and $N$. This result confirms the findings of [12]. However, it is worth observing that, due to the random nature of the process, the dynamics of convergence toward consensus is not necessarily monotonic as indicated in [12] and impasses, i.e., states $(s=(N / 2))$ where consensus is not reached, may emerge for specific configurations of the social space. Such impasses were overlooked in previous contributions. We provide a simple example showing how impasses can occur depending on the structure of the social space.

Example 3. Consider the case of 10 agents with two different social spaces: $\mathbf{n}_{1}=(3,3,4)$ and $\mathbf{n}_{2}=(5,5)$. Since at the end of each turn we have tables with homogeneous opinions, only states 0, 3, 4, 6, 7 and 10 are feasible in social space $\mathbf{n}_{1}$ and $0,5,10$ are feasible states in $\mathbf{n}_{2}$. It is clear that starting from states 0 and 1 the only possible evolution is to state 0 in both cases. As it concerns the other states, applying formula (3), we can compute the transition probability matrices:

$M_{\mathbf{n}_{1}}=\left[\begin{array}{ccccccccccc}1 & 0 & 0 & 0 & 0 & 0 & 0 & 0 & 0 & 0 & 0 \\ 1 & 0 & 0 & 0 & 0 & 0 & 0 & 0 & 0 & 0 & 0 \\ \frac{13}{15} & 0 & 0 & \frac{2}{15} & 0 & 0 & 0 & 0 & 0 & 0 & 0 \\ \frac{72}{120} & 0 & 0 & \frac{44}{120} & \frac{4}{120} & 0 & 0 & 0 & 0 & 0 & 0 \\ \frac{54}{210} & 0 & 0 & \frac{122}{210} & \frac{25}{210} & 0 & \frac{9}{210} & 0 & 0 & 0 & 0 \\ 0 & 0 & 0 & \frac{48}{84} & \frac{14}{84} & 0 & \frac{14}{84} & \frac{8}{84} & 0 & 0 & 0 \\ 0 & 0 & 0 & \frac{36}{210} & \frac{9}{210} & 0 & \frac{79}{210} & \frac{86}{210} & 0 & 0 & 0 \\ 0 & 0 & 0 & 0 & 0 & 0 & \frac{40}{120} & \frac{44}{120} & 0 & 0 & \frac{36}{120} \\ 0 & 0 & 0 & 0 & 0 & 0 & \frac{2}{15} & \frac{2}{15} & 0 & 0 & \frac{11}{15} \\ 0 & 0 & 0 & 0 & 0 & 0 & 0 & 0 & 0 & 0 & 1 \\ 0 & 0 & 0 & 0 & 0 & 0 & 0 & 0 & 0 & 0 & 1\end{array}\right] ; M_{\mathbf{n}_{2}}=\left[\begin{array}{cccccccccccc}1 & 0 & 0 & 0 & 0 & 0 & 0 & 0 & 0 & 0 & 0 \\ 1 & 0 & 0 & 0 & 0 & 0 & 0 & 0 & 0 & 0 & 0 \\ 1 & 0 & 0 & 0 & 0 & 0 & 0 & 0 & 0 & 0 & 0 \\ \frac{5}{6} & 0 & 0 & 0 & 0 & \frac{1}{6} & 0 & 0 & 0 & 0 & 0 \\ \frac{10}{21} & 0 & 0 & 0 & 0 & \frac{11}{21} & 0 & 0 & 0 & 0 & 0 \\ 0 & 0 & 0 & 0 & 0 & 1 & 0 & 0 & 0 & 0 & 0 \\ 0 & 0 & 0 & 0 & 0 & \frac{11}{21} & 0 & 0 & 0 & 0 & \frac{10}{21} \\ 0 & 0 & 0 & 0 & 0 & \frac{1}{6} & 0 & 0 & 0 & 0 & \frac{5}{6} \\ 0 & 0 & 0 & 0 & 0 & 0 & 0 & 0 & 0 & 0 & 1 \\ 0 & 0 & 0 & 0 & 0 & 0 & 0 & 0 & 0 & 0 & 1 \\ 0 & 0 & 0 & 0 & 0 & 0 & 0 & 0 & 0 & 0 & 1\end{array}\right]$.

In the first case, the dynamics is consistent to Galam's model prediction, see, e.g. [12]. By contrast, in the second case, as we have only two odd-size tables of the same size, the transition matrix has three absorbing states as pointed out in Theorem 2. Therefore the dynamics described by the Markov process is different from the one of [12].

Remark 4. In the transition matrices of the previous and the following examples the zero columns correspond to states that are not feasible, i.e. $\left(S / F_{\mathbf{n}}\right)$. Nevertheless, as these states can be the initial condition of the process, they are not omitted from our analysis. 
Obviously, even when considering a non-biased discussion function such as (5), absorbing states different from consensus states $(0$ and $N)$ are possible.

\section{Stochastic killing point and quantitative illustration}

Using the results of the previous section, we are able to analyze the diffusion of rumors. Indeed, given the initial distribution vector $\boldsymbol{\pi}_{0}$, the absorbing Markov chain $\left(M_{\mathbf{n}}, F_{\mathbf{n}}\right)$ describes the dynamics of rumors under majority rule in an $N$ person-population with social space $\mathbf{n}$. Once the absorbing states of the Markov chain have been determined, it is possible to analyze the process in more detail. In fact, by simple algebra it is possible to provide some important statistics, such as the expected time of convergence and the probability to reach consensus on each of the two opinions. However, in order to perform this analysis and to understand the rule of the social space in the convergence to consensus, it is first useful to define the equivalent of the so called killing point for the Markov chain $\left(M_{\mathbf{n}}, F_{\mathbf{n}}\right)$. In [12] the killing point is the point where the monotonic flow -towards one of two stable fixed points- changes direction. The killing point is important to characterize the rumor dynamics as it determines the border of the basin of attractions of the two stable fixed points 0 and 1.

To extend this notion to our formalization recall that now the fixed points 0 and 1 correspond to the absorbing states 0 and $N$. Focusing on the case of two absorbing states only, i.e. excluding the cases of social spaces made up of only two odd-sized tables of the same dimension, and denoting with $p_{i j}(t)$ the $t$-step transition probability, it is possible to define an analogue of the killing point for the absorbing Markov chain proposed here. Let us call it stochastic killing point.

Definition 5. Excluding the cases for which the stochastic matrix $M_{\mathbf{n}}$ is characterized by more than two absorbing states, we define the stochastic killing point of $\left(M_{\mathbf{n}}, F_{\mathbf{n}}\right)$ as $k^{*}=Y^{0}$, such that $\lim _{t \rightarrow \infty} p_{Y^{0}, 0}(t) \geq$ $\lim _{t \rightarrow \infty} p_{Y^{0}, N}(t)$ and $\lim _{t \rightarrow \infty} p_{Y^{0}+1,0}(t)<\lim _{t \rightarrow \infty} p_{Y^{0}+1, N}(t)$, with $Y^{0} \in S$.

The value of $k^{*}$ can be easily calculated. Let us rewrite the transition matrix in the normal form:

$$
\bar{M}_{\mathbf{n}}=\left[\begin{array}{cc}
I_{\mathbf{n}} & 0 \\
R_{\mathbf{n}} & Q_{\mathbf{n}}
\end{array}\right],
$$

where $I_{\mathbf{n}}$ is the identity matrix identifying the absorbing states related to the social space $\mathbf{n}$, in our case 0 and $N, Q_{\mathbf{n}}$ gives the transition probabilities between transient states and $R_{\mathbf{n}}$ transition probabilities to absorbing states. We compute the fundamental matrix ${ }^{3} Z_{\mathbf{n}}=\left[I_{\mathbf{n}}^{\prime}-Q_{\mathbf{n}}\right]^{-1}$ and $B_{\mathbf{n}}=Z_{\mathbf{n}} R_{\mathbf{n}}$, where $I_{\mathbf{n}}^{\prime}$ is the identity matrix identifying the transient states related to $\mathbf{n}$ and $B_{\mathbf{n}}(i, j)$ indicates the probability to end up

\footnotetext{
${ }^{3}$ In this section, some classical results on absorbing Markov chains are used without explanation, for a general treatment see [15].
} 
at the absorbing state $j$ when starting from transient state $i$. Since we are considering transition matrices with two absorbing states $(0$ and $N), B_{\mathbf{n}}$ has two columns. Let us introduce the following matrix

$$
\bar{B}_{\mathbf{n}}=\left[\begin{array}{c}
{[1,0]} \\
B_{\mathbf{n}} \\
{[0,1]}
\end{array}\right],
$$

where entries $b_{1, j}$ (with $\left.j=0, \ldots N\right)$ give the probability that state $j$ will be absorbed in state 0 , while entries $b_{2, j}$ give the probability that state $j$ will be absorbed in state $N, \forall j \in S$. Under the majority rule dynamics, $b_{1, j}$ is monotonically non-increasing with respect to $j$ and $b_{2, j}$ is monotonically non-decreasing with respect to $j$, therefore the stochastic killing point is $k^{*}=\widehat{j}$ where $\widehat{j}$ is such that $b_{1, \widehat{j}}>b_{2, \widehat{j}}$ and $b_{1, \widehat{j}+1}<b_{2, \widehat{j}+1}$.

The stochastic killing point is important to understand the effects of different social spaces on the probability of reaching consensus either for opinion '-' or ' + '. We report some examples which illustrate this aspect providing the average $\rho=N / L$ and the variance $\sigma^{2}=\sum_{T_{r} \in \mathcal{N}}\left(\left|T_{r}\right|-(N / L)\right)^{2}$ of the size of the tables of the considered social spaces. In particular, let us consider a population of 10 agents as in Example 3 with social space $\mathbf{n}_{1}$ and two additional ones, $\mathbf{n}_{3}=(1,1,1,1,1,1,1,1,2)$ and $\mathbf{n}_{4}=(1,1,8)$. For each social space, we compute the stochastic matrix $M_{\mathbf{n}}$ in normal form, vector $\left(z_{0}, \ldots, z_{j}, \ldots, z_{N}\right)^{\mathrm{T}}$, where $z_{0}=z_{N}=0$ and $\left(z_{1}, \ldots, z_{j}, \ldots, z_{N-1}\right)^{\mathrm{T}}=Z_{\mathbf{n}} \mathbf{1}-1$, and matrix $\bar{B}_{\mathbf{n}}$. These cases are represented in Figures 2,3 and 4 . The left side of these figures represents the probabilities $b_{1, j}$ and $b_{2, j}$ to converge, starting from state $j \in S$, respectively to absorbing states 0 , and $N$. Probabilities for state 0 , i.e., consensus on opinion '-', are depicted with black dots; those for state $N$, i.e., consensus on opinion ' + ', are depicted with gray dots. The right side of the figures represents the expected time, $z_{j}$, of convergence to consensus (either 0 or $N$ ) for each initial state $j \in S$. As we are considering discrete states the dashed lines are there for illustrative purpose only. The caption of each figure also provides the average $\rho$ and the variance $\sigma$ of the size of the tables in the social space.

In the following, we show how the average and the variance of the size of the tables in the social space affect the diffusion of rumors.

In Figures 2 and 3 we consider two social spaces with the same $N$ and similar in terms of $\sigma^{2}$. As $\rho$ increases from $\rho=10 / 9$ to $\rho=10 / 3$ the stochastic killing point shifts to the left (compare Figures 2 a) and $3 \mathrm{a})$ ), and the expected time required to reach consensus decreases, (compare Figures $2 \mathrm{~b}$ ) and $3 \mathrm{~b}$ )).

The interpretation is straightforward: an initial minority supporting the rumor has less probability to influence the majority (which does not support the rumor) and to change the common opinion about a fact when the discussion takes place in large groups (large $\rho$ ). In other words, encouraging debate with many people serves as a deterrent for the diffusion of rumors. 

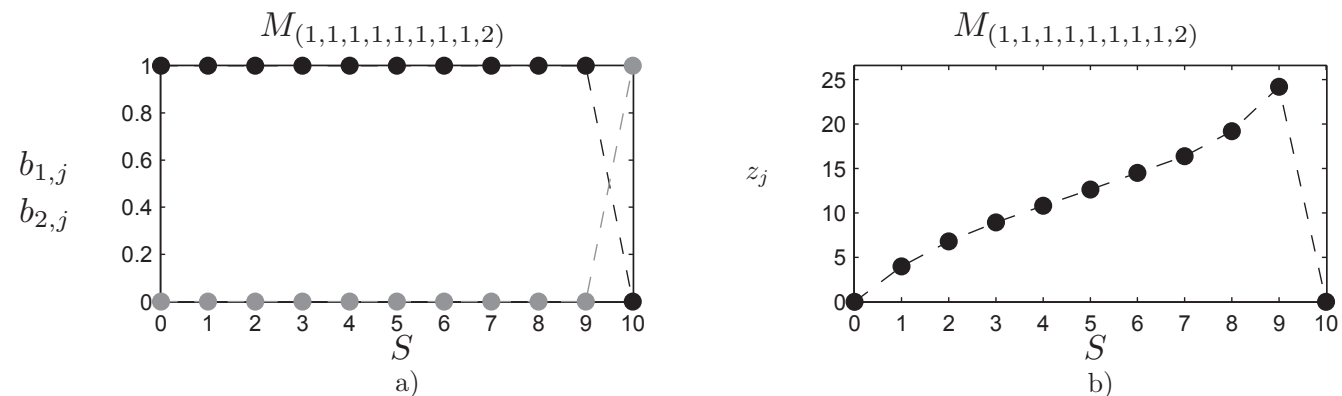

b)

Figure 2: Case $\mathbf{n}_{3}=(1,1,1,1,1,1,1,1,2), \rho=10 / 9, \sigma^{2} \approx 0.8889$ and $k^{*}=9$. Depending on the initial state $j \in S:$ a) probability to converge to the absorbing states 0 (black circles) and $N$ (gray circles); b) expected time of convergence to consensus (either 0 or $N$ ).
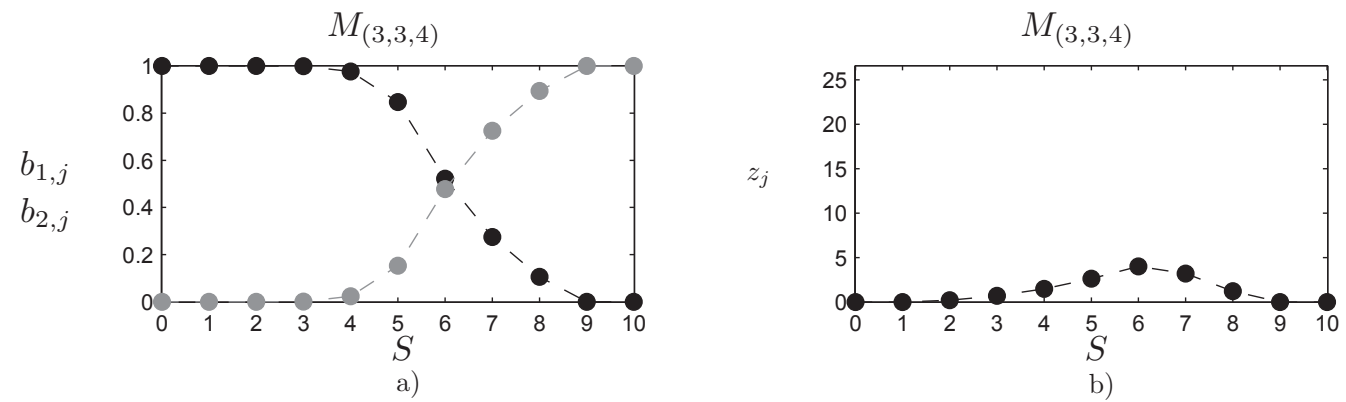

Figure 3: Case $\mathbf{n}_{1}=(3,3,4), \rho=10 / 3, \sigma^{2} \approx 0.6667$ and $k^{*}=6$. Depending on the initial state $j \in S:$ a) probability to converge to the absorbing states 0 (black circles) and $N$ (gray circles); b) expected time of convergence to consensus (either 0 or $N)$.

A similar effect is observed increasing $\sigma^{2}$ and keeping $\rho$ constant. Considering two social spaces with the same $N$ and $\rho$, increasing $\sigma^{2}$, i.e., people gather either in very large groups or very small groups, the stochastic killing point tends to shift to the left, compare Figures 3 a) $\left(\sigma^{2} \approx 0.6667\right)$ and 4 a) $\left(\sigma^{2} \approx 32.6667\right)$. This means that the possibility that the rumor spreads across the population decreases and the expected time of convergence to consensus reduces, compare Figures 3 b) and 4 b). These examples suggest that the larger the average $\rho$ and the variance $\sigma^{2}$ of the size of the tables, the lower the probability for a rumor to spread across the population. The reason for this is that when $\rho$ and $\sigma^{2}$ are large, interactions among agents take place in few large tables and the effect of the majority rule, i.e. the assumed propensity of agents to believe in rumors when at a table agents with opposing opinions match in number, has a lower weight. More generally, when the tables are large, the weight of an agent in influencing others reduces and the opinion of the majority prevails on the one of the minority more easily. The analysis we conducted suggests that, when discussion takes place in large groups, the majority rule works well and prevents the diffusion of rumors. 


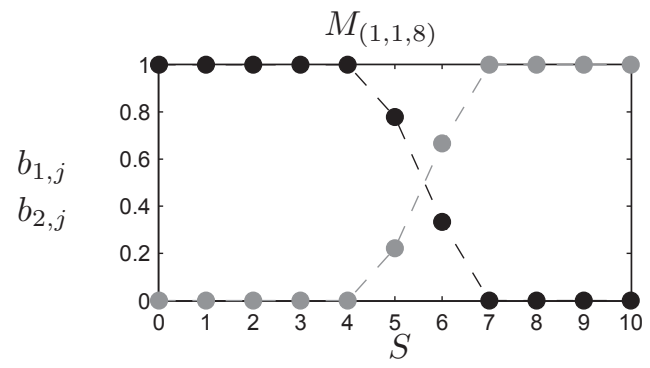

a)

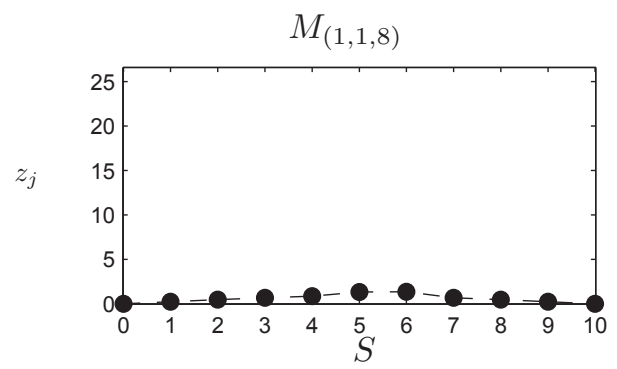

b)

Figure 4: Case $\mathbf{n}_{4}=(1,1,8), \rho=10 / 3, \sigma^{2} \approx 32.6667$ and $k^{*}=5$. Depending on the initial state $j \in S$ : a) probability to converge to the absorbing states 0 (black circles) and $N$ (gray circles); b) expected time of convergence to consensus (either 0 or $N)$.

\section{Conditional expected value}

In section 2 we proved that the stochastic process $\left(Y^{t}\right)_{t \geq 0}$ describing the dynamics of rumors under majority rule dynamic is an absorbing Markov chain. In this section we show that, given the state of the process at time $t$, it is possible to calculate the conditional expected value of the process at time $t+1$. Here we provide a closed form solution for $E\left[Y^{t+1} \mid Y^{t}=y\right]$. To this purpose, let us define the set $\omega_{i, T_{r}^{+}} \subseteq \Omega_{i}$ as

$$
\omega_{i, T_{r}^{+}}=\left\{\mathbf{y}: \mathbf{y} \in \Omega_{y} \text { and } y_{r} \geq\left\lfloor\frac{\left|T_{r}\right|}{2}\right\rfloor+1\right\} \text {. }
$$

This is the event "given $y$ agents with opinion ' + ', at table $T_{r}$ the majority of agents have opinion ' + ' ". Moreover, let us provide the following results:

Lemma 6. Given the absorbing Markov chain $\left(M_{\mathbf{n}}, F_{\mathbf{n}}\right)$ and the probability measure $P_{y}(\mathbf{y}) \forall y \in S$ and $\forall \mathbf{y} \in \Omega_{y}$, then

$$
G\left(\omega_{i, T_{r}^{+}}\right)=\sum_{y_{r}=\left\lfloor\frac{\left|T_{r}\right|}{2}\right\rfloor+1}^{\min \left(\left|T_{r}\right|, y\right)} \frac{\left(\begin{array}{c}
\left|T_{r}\right| \\
y_{r}
\end{array}\right)\left(\begin{array}{c}
N-\left|T_{r}\right| \\
y-y_{r}
\end{array}\right)}{\left(\begin{array}{c}
N \\
y
\end{array}\right)}
$$

is the probability of the event $\omega_{i, T_{r}^{+}}$.

Proof. According to Proposition 1, the probability of the event $\omega_{i, T_{r}^{+}}$is

$$
G\left(\omega_{i, T_{r}^{+}}\right)=\sum_{\mathbf{y} \in \omega_{i, T_{r}^{+}}} P(\mathbf{y})=\sum_{\mathbf{y} \in \omega_{i, T_{r}^{+}}} \frac{\left(\begin{array}{c}
y \\
y_{1}, \ldots, y_{L}
\end{array}\right)\left(\begin{array}{c}
N-y \\
\left|T_{1}\right|-y_{1}, \ldots,\left|T_{L}\right|-y_{L}
\end{array}\right)}{N}
$$

which in an explicit form becomes:

$$
G\left(\omega_{i, T_{r}^{+}}\right)=\sum_{y_{r}=\left\lfloor\frac{\left|T_{r}\right|}{2}\right\rfloor+1}^{\min \left(\left|T_{r}\right|, y\right)}\left[\sum_{\begin{array}{c}
y_{1} \\
y_{1}, \ldots, y_{r-1}, y_{r+1}, \ldots, y_{L}: \\
y_{1}+\ldots+y_{r-1}+y_{r+1}+\ldots+y_{L}=y-y_{r}
\end{array}} \frac{\left.\begin{array}{c}
N-y \\
y_{1}, \ldots, y_{N}
\end{array}\right)\left(\begin{array}{c}
N\left|T_{1}\right|-y_{1}, \ldots,\left|T_{L}\right|-y_{L} \\
N
\end{array}\right)}{\left(\begin{array}{c}
N \\
\left|T_{L}\right|, \ldots,\left|T_{L}\right|
\end{array}\right)}\right] .
$$


Multiplying and dividing by $\left(\begin{array}{c}N-n_{r} \\ y-y_{r}\end{array}\right)$ and after some rearrangements, it is easy to obtain:

$$
G\left(\omega_{i, T_{r}^{+}}\right)=\sum_{y_{r}=\left\lfloor\frac{\left|T_{r}\right|}{2}\right\rfloor+1}^{\min \left(\left|T_{r}\right|, y\right)} \frac{\left(\begin{array}{c}
\left|T_{r}\right| \\
y_{r}
\end{array}\right)\left(\begin{array}{c}
N-\left|T_{r}\right| \\
y-y_{r}
\end{array}\right)}{\left(\begin{array}{c}
N \\
y
\end{array}\right)}\left[\sum_{\begin{array}{c}
y_{1}, \ldots, y_{r-1}, y_{r+1}, \ldots, y_{L}: \\
y_{1}+\ldots+y_{r-1}+y_{r+1}+\ldots+y_{L}=y-y_{r}
\end{array}} \frac{\left(\begin{array}{c}
\left|T_{1}\right| \\
y_{1}
\end{array}\right) \cdots\left(\begin{array}{c}
\left|T_{r-1}\right| \\
y_{r-1}
\end{array}\right)\left(\begin{array}{c}
\left|T_{r+1}\right| \\
y_{r+1}
\end{array}\right) \cdots\left(\begin{array}{c}
\left|T_{L}\right| \\
y_{L}
\end{array}\right)}{\left(\begin{array}{c}
N-\left|T_{r}\right| \\
y-y_{r}
\end{array}\right)}\right],
$$

where the quantity inside the brackets is equal to 1 by Chu-Vandermonde's identity. From which the claim follows.

It is worth noting that $G\left(\omega_{i, T_{r}^{+}}\right)=G\left(\omega_{i, T_{q}^{+}}\right)$if $T_{r}, T_{q} \in \mathcal{N}$ and $\left|T_{r}\right|=\left|T_{q}\right|$. Using the results of the previous lemma and given $Y^{t}=y$, it is possible to derive the conditional expected value for the random variable $Y^{t+1}$.

Proposition 7. Given the absorbing Markov chain $\left(M_{\mathbf{n}}, F_{\mathbf{n}}\right)$ and $Y^{t}=y$, the conditional expected value of the random variable $Y^{t+1}$ is

$$
\mathrm{E}\left[Y^{t+1} \mid Y^{t}=y\right]=N \sum_{k=1}^{K} a_{k} \sum_{j=\left\lfloor\frac{k}{2}\right\rfloor+1}^{\min (k, y)} \frac{\left(\begin{array}{l}
k \\
j
\end{array}\right)\left(\begin{array}{l}
N-k \\
y-j
\end{array}\right)}{\left(\begin{array}{l}
N \\
y
\end{array}\right)}
$$

where $K$ is the number of seats of the larger table of the social space $\mathcal{N}$.

Proof. The conditional expected value of $Y^{t+1}$ can be easily written as

$$
\mathrm{E}\left[Y^{t+1} \mid Y^{t}=y\right]=\sum_{T_{r} \in \mathcal{N}}\left|T_{r}\right| G\left(\omega_{i, T_{r}^{+}}\right) .
$$

Collecting the same size tables by (1) and let $G\left(\omega_{i, T_{r}^{+}}\right)=G\left(\omega_{i, k^{+}}\right)$if $\left|T_{r}\right|=k$, we have

$$
\mathrm{E}\left[Y^{t+1} \mid Y^{t}=y\right]=\sum_{k=1}^{K} k \tau_{k} G\left(\omega_{i, k^{+}}\right)
$$

which can be rewritten as follows

$$
\mathrm{E}\left[Y^{t+1} \mid Y^{t}=y\right]=N \sum_{k=1}^{K} \frac{k}{N} \tau_{k} G\left(\omega_{i, k^{+}}\right)
$$

and by (2) and Lemma 6 the thesis follows.

The closed form solution of the conditional expected value of the stochastic process is particularly useful for calibrations and can be used as an alternative to Galam's formula when the social space is large and the calculations to obtain the transition matrix are cumbersome.

\section{Conclusion}

In this paper, we propose an exhaustive formulation for rumors spreading described in [12]. Specifically, the paper shows that the diffusion of rumors follows an absorbing Markov chain which partially confirms the 
results provided in [12].

The mathematical formulation of the model we propose is particularly suitable for studying the diffusion of opinions in small communities. When the number of people in the communities is relatively small the calculations required to obtain the transition matrix are still tractable and a complete description of the phenomena can be provided. For bigger groups of people, the transition matrix requires a cumbersome calculation to be defined and the conditional expected values derived in Proposition 7 can be used as an alternative to the Galam's formula to obtain a prediction for the dynamics of the phenomena.

Our approach extends Galam's analysis providing further results and precision. Using a Markov chain helps to have further information about the process of diffusion of opinions which are not possible to obtain using the original Galam's formulation. In particular, it is possible to compute the expected time of absorption for each transient state and the probability to reach a specific absorbing state starting from a transient state. Finally, using the more precise formalization that we provide, it is possible to prove the existence of impasses, i.e., absorbing states different from 0 and $N$, which were impossible to detect with the original formalization. Moreover, the approach we propose is also useful for other reasons. First of all, it provides some consistency to existing literature on opinion dynamics, such as the DeGroot type models, as we illustrate that even Galam's model can be formalized as Markov chains. Secondly, formulating Galam's model as a Markov process provides a starting point to compare Galam's and DeGroot's models and to understand their differences. In particular, contributions such as [10] are steps in this direction, as their approach provides a flexible tool to model people inclinations towards two binary choices "yes" or "no" and to transform a discrete opinion model in a continuum of opinions. The framework provided in [10] is so general that it can even be used to model an inclination of agents towards the opinion of the minority. However, the gap between the two approaches is still not completely closed as the subgroup structure of the Galam's model is an essential feature to explain the phenomenon of minority opinion spreading that is not considered in [10].

With further research it will be possible to extend the analysis in several directions. First of all, starting from the formalization we provide, it would be interesting to analyze the role that the social space has on the final outcome ${ }^{4}$, then some of the assumptions can be relaxed in order to make the model more realistic, for example modeling individual influence and introducing a more complex-network structure for connecting individuals as in [1]. In addition, it would be interesting to assume that the process by which individuals reach consensus within the group is similar to the one considered in [9]. Moving further on in this direction, another line of research is modeling interactions other than discussions. For example, in [5], the

\footnotetext{
${ }^{4}$ We are grateful to an anonymous referee for this suggestion.
} 
interactions among agents may consist of binary choices (see, for instance, Schelling's seminal contribution [19]) as formalized in [4]. Yet, binary choices are just one possibility among the others, as several other interactions can be considered, see for example [8] and references therein. Finally, it will be interesting to analyze the dinamics when some individuals dismiss opposite opinions no matter how valid as this will prevent full polarization and would be more realistic.

\section{Acknowledgements}

The authors would like to thank Gian-Italo Bischi, Herbert Dawid, Tim Hellmann and three anonymous referees for their helpful comments. Furthermore, the authors are grateful to the participants of the 2011 conference entitled "Unexpected Conference. SOCIOPHYSICS: Do humans behave like atoms?", of the 2012 annual meeting of Italian Association for Mathematics Applied to Economic and Social Sciences (A.M.S.E.S.), of the 2012 BiGSEM Colloquium (Bielefeld University) and of the 2013 seminars of the University of Urbino "Carlo Bo" and to the 2013 seminars of the University of Calabria for helpful discussions. This work has been performed under the auspices of COST Action IS1104 "The EU in the new complex geography of economic systems: models, tools and policy evaluation". The usual caveats apply.

\section{Appendix}

Proof of Theorem 2. By Proposition 1 and the transition probability formula, it is clear that $\left(M_{\mathbf{n}}, F_{\mathbf{n}}\right)$ is a Markov chain and states 0 and $N$ are always absorbing, i.e. $p_{0,0}=1$ and $p_{N, N}=1$. In the following, we will prove that there is at most another absorbing state and all the other states are transient.

As we have mentioned above, the same state $v$ may be the result of reaching the majority at different combinations of tables. Let us denote $\mathcal{C}_{v}$ the set of all the table combinations giving state $v$.

In order to investigate the existence of other possible absorbing states different from 0 and $N$, let us consider an arbitrary feasible state $v\left(\in F_{\mathbf{n}}\right)$ such that $v \neq 0$ and $v \neq N$. Consider an arbitrary $C_{v} \in \mathcal{C}_{v}$ and other two states $u$ and $w$, such that:

$$
u=\sum_{T_{r} \in C_{v}}\left|T_{r}\right|-\left|T_{q}\right|<\sum_{T_{r} \in C_{v}}\left|T_{r}\right|=v<\sum_{T_{r} \in C_{v}}\left|T_{r}\right|+\left|T_{z}\right|=w,
$$

where $T_{q}$ is the minimal size table in $C_{v}$, and $T_{z}$ is the minimal size table in $\left(\mathcal{N} / C_{v}\right)$. It is clear that $u, w \in F_{\mathbf{n}}$, i.e., they are feasible states.

These table combinations are illustrated in Figure .5; the gray regions respectively represent $\mathcal{N} / C_{u}$, $\mathcal{N} / C_{v}$ and $\mathcal{N} / C_{w}$. 


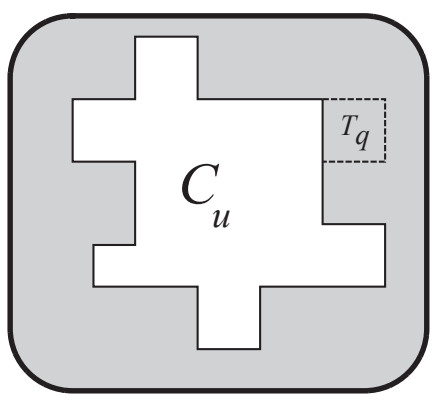

a)

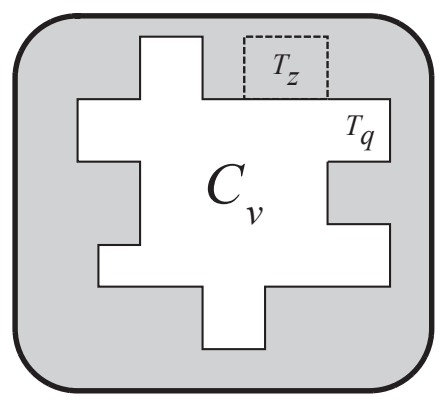

b)

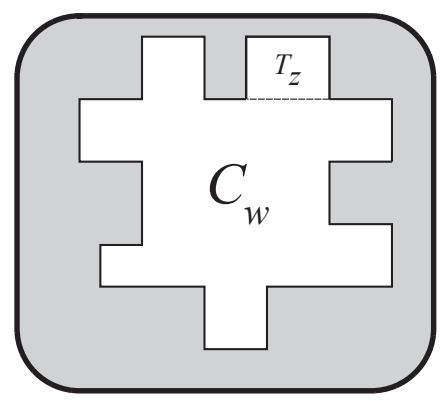

c)

Figure .5: Table combinations for states $u<v<w:$ a) $C_{u}$, b) $C_{v}$, and c) $C_{w}$.

If $v$ is an absorbing state then it must be $p_{v, u}=p_{v, w}=0$; we will show that this cannot hold and therefore $v$ cannot be an absorbing state ${ }^{5}$.

Let us consider $p_{v, u}=0$, this means that from state $v$ it is impossible to reach state $u$, that is, for all the table combinations $C_{u} \in \mathcal{C}_{u}$, relative to state $u$, it is impossible that the majority of opinion ' + ' is reached only at the tables in $C_{u}$. This means that even if all the seats at tables in $C_{u}$ are taken by the $v$ agents with opinion ' + ', the remaining $v-u$ agents with opinion ' + ' cannot be seated at tables in $\mathcal{N} / C_{u}$, without reaching the majority on at least one of these tables ${ }^{6}$. Formally

$$
v-u \geq 1+\sum_{T_{r} \in\left(\mathcal{N} / C_{u}\right)}\left\lfloor\frac{\left|T_{r}\right|}{2}\right\rfloor,
$$

where $\sum_{T_{r} \in\left(\mathcal{N} / C_{u}\right)}\left\lfloor\frac{\left|T_{r}\right|}{2}\right\rfloor$ is the maximum number of agents that can be seated at tables in $\mathcal{N} / C_{u}$ without reaching the majority of opinion ' + '. As $\left|T_{q}\right|=v-u$, Equation (.1) can be rewritten as

$$
\left|T_{q}\right| \geq 1+\sum_{T_{r} \in\left(\mathcal{N} / C_{u}\right)}\left\lfloor\frac{\left|T_{r}\right|}{2}\right\rfloor .
$$

From Figure .5 a) and b), we can see that, table combinations giving states $v$ and $u$ differ by table $T_{q}$ only. Therefore, it follows that $\mathcal{N} / C_{u}=\left\{T_{q}\right\} \cup \mathcal{N} / C_{v}$ and that, in order not to reach the majority of opinion ' + ' at tables in $\mathcal{N} / C_{u}$, such a majority must not be reached neither at table $T_{q}$ nor at tables in $\mathcal{N} / C_{v}$, that is $\sum_{T_{r} \in\left(\mathcal{N} / C_{u}\right)}\left\lfloor\frac{\left\lfloor T_{r} \mid\right.}{2}\right\rfloor=\left\lfloor\frac{\left.\mid T_{q}\right\rfloor}{2}\right\rfloor+\sum_{T_{r} \in\left(\mathcal{N} / C_{v}\right)}\left\lfloor\frac{\left|T_{r}\right|}{2}\right\rfloor$. As a consequence Equation (.2) can be rewritten as:

$$
\left|T_{q}\right| \geq 1+\left\lfloor\frac{\left|T_{q}\right|}{2}\right\rfloor+\sum_{T_{r} \in\left(\mathcal{N} / C_{v}\right)}\left\lfloor\frac{\left|T_{r}\right|}{2}\right\rfloor .
$$

Let us consider the table combinations giving states $u<v<w$; we already know $\sum_{T_{r} \in\left(\mathcal{N} / C_{u}\right)}\left\lfloor\frac{\left\lfloor T_{r}\right\rfloor}{2}\right\rfloor=$

\footnotetext{
${ }^{5}$ Therefore, to prove that $v$ is not an absorbing state, it will be sufficient to see that, for an arbitrary $C_{v} \in \mathcal{C}_{v}$, holds either $p_{v, u} \neq 0$ or $p_{v, w} \neq 0$.

${ }^{6}$ Obviously, this condition must hold $\forall C_{u} \in \mathcal{C}_{u}$.
} 
$\left\lfloor\frac{\left|T_{q}\right|}{2}\right\rfloor+\sum_{T_{r} \in\left(\mathcal{N} / C_{v}\right)}\left\lfloor\frac{\left|T_{r}\right|}{2}\right\rfloor$. Furthermore, $\left\lfloor\frac{\left|T_{z}\right|}{2}\right\rfloor+\sum_{T_{r} \in\left(\mathcal{N} / C_{w}\right)}\left\lfloor\frac{\left|T_{r}\right|}{2}\right\rfloor=\sum_{T_{r} \in\left(\mathcal{N} / C_{v}\right)}\left\lfloor\frac{\left|T_{r}\right|}{2}\right\rfloor$ as $T_{z} \in\left(\mathcal{N} / C_{v}\right) ;$ therefore, (.3) can be rewritten as

$$
\left|T_{q}\right| \geq 1+\left\lfloor\frac{\left|T_{q}\right|}{2}\right\rfloor+\left\lfloor\frac{\left|T_{z}\right|}{2}\right\rfloor+\sum_{T_{r} \in\left(\mathcal{N} / C_{w}\right)}\left\lfloor\frac{\left|T_{r}\right|}{2}\right\rfloor .
$$

We can observe that if $\left|T_{q}\right|<\left|T_{z}\right|$, (.4) does not hold. Now let us examine the two remaining cases $\left|T_{q}\right|>\left|T_{z}\right|$ and $\left|T_{q}\right|=\left|T_{z}\right|$ separately.

1. $\left|T_{q}\right|>\left|T_{z}\right|$. Consider the feasible state $u^{\prime}=u+\left|T_{z}\right|<v$; taking into account the table combinations we have

$$
u^{\prime}=\sum_{T_{r} \in C_{u}}\left|T_{r}\right|+\left|T_{z}\right|=\sum_{T_{r} \in C_{v}}\left|T_{r}\right|-\left|T_{q}\right|+\left|T_{z}\right|<\sum_{T_{r} \in C_{v}}\left|T_{r}\right|=v,
$$

as $\left|T_{q}\right|>\left|T_{z}\right|$. Obviously if $v$ is an absorbing state, then it must also be $p_{v, u^{\prime}}=0$.

If $p_{v, u^{\prime}}=0$ then, for all $C_{u^{\prime}} \in \mathcal{C}_{u^{\prime}}$, after $u^{\prime}$ agents are seated at the tables in $C_{u^{\prime}}$, the majority is reached on at least one of the tables in $\mathcal{N} / C_{u^{\prime}}$, and therefore

$$
v-u^{\prime}=\left|T_{q}\right|-\left|T_{z}\right| \geq 1+\sum_{T_{r} \in\left(\mathcal{N} / C_{u^{\prime}}\right)}\left\lfloor\frac{\left|T_{r}\right|}{2}\right\rfloor,
$$

which can be rewritten as:

$$
\left|T_{q}\right|-\left|T_{z}\right| \geq 1+\sum_{T_{r} \in\left(\mathcal{N} / C_{u^{\prime}}\right)}\left\lfloor\frac{\left|T_{r}\right|}{2}\right\rfloor=1+\left\lfloor\frac{\left|T_{q}\right|}{2}\right\rfloor-\left\lfloor\frac{\left|T_{z}\right|}{2}\right\rfloor+\sum_{T_{r} \in\left(\mathcal{N} / C_{v}\right)}\left\lfloor\frac{\left|T_{r}\right|}{2}\right\rfloor .
$$

Since $T_{z} \in\left(\mathcal{N} / C_{v}\right)$, it holds $\sum_{T_{r} \in\left(\mathcal{N} / C_{v}\right)}\left\lfloor\frac{\left|T_{r}\right|}{2}\right\rfloor>\left\lfloor\frac{\left|T_{z}\right|}{2}\right\rfloor$, and we have:

$$
\left|T_{q}\right|-\left|T_{z}\right| \geq 1+\left\lfloor\frac{\left|T_{q}\right|}{2}\right\rfloor-\left\lfloor\frac{\left|T_{z}\right|}{2}\right\rfloor+\sum_{T_{r} \in\left(\mathcal{N} / C_{v}\right)}\left\lfloor\frac{\left|T_{r}\right|}{2}\right\rfloor \geq 1+\left\lfloor\frac{\left|T_{q}\right|}{2}\right\rfloor .
$$

Now consider state $w$; as $v$ is assumed to be an absorbing state, it must be $p_{v, w}=0$ as well.

If $p_{v, w}=0$ then, for all $C_{w} \in \mathcal{C}_{w}, v$ is smaller than the minimal number of agents with opinion ' + ' to have the majority at each of the tables in $C_{w}=C_{v} \cup\left\{T_{z}\right\}$. Formally:

$$
v=\sum_{T_{r} \in C_{v}}\left|T_{r}\right|<\sum_{T_{r} \in C_{w}}\left(\left\lfloor\frac{\left|T_{r}\right|}{2}\right\rfloor+1\right)=\left(\left\lfloor\frac{\left|T_{z}\right|}{2}\right\rfloor+1\right)+\sum_{T_{r} \in C_{v}}\left(\left\lfloor\frac{\left|T_{r}\right|}{2}\right\rfloor+1\right) .
$$

Rearranging some terms, condition (.7) can be rewritten as

$$
\sum_{T_{r} \in C_{v}}\left|T_{r}\right|-\sum_{T_{r} \in C_{v}}\left(\left\lfloor\frac{\left|T_{r}\right|}{2}\right\rfloor+1\right)<\left(\left\lfloor\frac{\left|T_{z}\right|}{2}\right\rfloor+1\right),
$$

which is equivalent to

$$
\sum_{T_{r} \in C_{v}}\left(\left|T_{r}\right|-\left\lfloor\frac{\left|T_{r}\right|}{2}\right\rfloor-1\right)<\left\lfloor\frac{\left|T_{z}\right|}{2}\right\rfloor+1
$$


Since $T_{q} \in C_{v}$, the condition (.9) can be rewritten as

$$
\left|T_{q}\right|-\left\lfloor\frac{\left|T_{q}\right|}{2}\right\rfloor-1+\sum_{T_{r} \in C_{u}}\left(\left|T_{r}\right|-\left\lfloor\frac{\left|T_{r}\right|}{2}\right\rfloor-1\right)<\left\lfloor\frac{\left|T_{z}\right|}{2}\right\rfloor+1 .
$$

From $p_{v, u^{\prime}}=0$ and $p_{v, w}=0$ respectively follow conditions (.6) and (.10); now we show that from these latter conditions a contradiction follows. In fact, condition (.6) can be rewritten as $-\left|T_{q}\right|+\left|T_{z}\right| \leq$ $-\left\lfloor\frac{\left.\mid T_{q}\right\rfloor}{2}\right\rfloor-1$, which plugged in (.10) gives:

$$
\left|T_{q}\right|-\left|T_{q}\right|+\left|T_{z}\right|+\sum_{T_{r} \in C_{u}}\left(\left|T_{r}\right|-\left\lfloor\frac{\left|T_{r}\right|}{2}\right\rfloor-1\right)<\left\lfloor\frac{\left|T_{z}\right|}{2}\right\rfloor+1
$$

which can be simplified to

$$
\left|T_{z}\right|+\sum_{T_{r} \in C_{u}}\left(\left|T_{r}\right|-\left\lfloor\frac{\left|T_{r}\right|}{2}\right\rfloor-1\right)<\left\lfloor\frac{\left|T_{z}\right|}{2}\right\rfloor+1 .
$$

Since $\sum_{T_{r} \in\left(C_{v} /\left\{T_{q}\right\}\right)}\left(\left|T_{r}\right|-\left\lfloor\frac{\left|T_{r}\right|}{2}\right\rfloor-1\right) \geq 0$ and $\left|T_{z}\right| \geq\left\lfloor\frac{\left|T_{z}\right|}{2}\right\rfloor+1$, it follows that (.12) is never satisfied. Therefore, conditions (.6) and (.10) cannot both hold at the same time and then, in this case, $v$ cannot be an absorbing state.

2. $\left|T_{q}\right|=\left|T_{z}\right|$. When $\left|T_{q}\right|$ and $\left|T_{z}\right|$ are even, condition (.4) cannot hold. On the other hand, when $\left|T_{q}\right|$ and $\left|T_{z}\right|$ are odd, from condition (.4) it follows that

$$
\sum_{T_{r} \in\left(\mathcal{N} / C_{w}\right)}\left\lfloor\frac{\left|T_{r}\right|}{2}\right\rfloor=0 .
$$

Furthermore, rearranging terms in condition (.10) we obtain

$$
\left|T_{q}\right|-\left\lfloor\frac{\left|T_{q}\right|}{2}\right\rfloor-\left\lfloor\frac{\left|T_{z}\right|}{2}\right\rfloor-1+\sum_{T_{r} \in C_{u}}\left(\left|T_{r}\right|-\left\lfloor\frac{\left|T_{r}\right|}{2}\right\rfloor-1\right)<1 .
$$

As, in this case, $T_{q}$ and $T_{z}$ are two same odd size tables, it holds $\left|T_{q}\right|=\left\lfloor\frac{\left|T_{q}\right|}{2}\right\rfloor+\left\lfloor\frac{\left|T_{q}\right|}{2}\right\rfloor+1=$ $\left\lfloor\frac{\left.\mid T_{q}\right\rfloor}{2}\right\rfloor+\left\lfloor\frac{\left\lfloor T_{z}\right\rfloor}{2}\right\rfloor+1$, and therefore (.14) becomes

$$
\sum_{T_{r} \in C_{u}}\left(\left|T_{r}\right|-\left\lfloor\frac{\left|T_{r}\right|}{2}\right\rfloor-1\right)<1 .
$$

As $\sum_{T_{r} \in C_{u}}\left(\left|T_{r}\right|-\left\lfloor\frac{\left|T_{r}\right|}{2}\right\rfloor-1\right) \geq 0$, from condition (.15) it follows:

$$
\sum_{T_{r} \in C_{u}}\left(\left|T_{r}\right|-\left\lfloor\frac{\left|T_{r}\right|}{2}\right\rfloor-1\right)=0 .
$$

This condition holds if and only if all tables in $C_{u}$ have size not larger than 2 .

Let us discuss (.13) and (.16) first when both $C_{v}$ and $\mathcal{N} / C_{v}$ are nonempty sets and then when both $C_{v}$ and $\mathcal{N} / C_{v}$ are empty sets: 
(a) In the first case we can distinguish between two situations (remember that by assumption $T_{q}$ and $T_{z}$ are the smaller size tables in $C_{v}$ and $\left(\mathcal{N} / C_{v}\right)$ respectively):

i. Let us consider the case $\left|T_{q}\right|=\left|T_{z}\right|=1$; as we rule out the trivial case discussed in Footnote 1 , there exists at least a table $T_{g} \in \mathcal{N}$ such that $\left|T_{g}\right| \geq 2$. If $T_{g} \in \mathcal{N} / C_{v}$, then $T_{g} \in \mathcal{N} / C_{w}$ as $\left|T_{z}\right|=1$; therefore, condition (.13) cannot hold. On the other hand, if $T_{g} \in C_{v}$, then $T_{g} \in C_{u}$ as $\left|T_{q}\right|=1$. Since condition (.16) holds if and only if all the tables in $C_{u}$ have a size not larger than 2, it must be $\left|T_{g}\right|=2$. We show that even in such a case $v$ cannot be an absorbing state. Indeed, consider table combination $C_{u}^{\prime}=\left(C_{u} \cup\left\{T_{q}\right\} \cup\left\{T_{z}\right\}\right) /\left\{T_{g}\right\}$; as $\left|T_{g}\right|=\left|T_{z}\right|+\left|T_{q}\right|$ it follows that $C_{u}^{\prime} \in \mathcal{C}_{u}$. We showed that, if $p_{v u}=0$, then for all $C_{u} \in \mathcal{C}_{u}$ condition (.2) holds. In particular, considering $C_{u}^{\prime} \in \mathcal{C}_{u}$ we have

$$
\left|T_{q}\right|=v-u \geq 1+\sum_{T_{r} \in\left(\mathcal{N} / C_{u}^{\prime}\right)}\left\lfloor\frac{\left|T_{r}\right|}{2}\right\rfloor .
$$

As $T_{g} \notin C_{u}^{\prime}$ it follows that $T_{g} \in \mathcal{N} / C_{u}^{\prime}$ and

$$
\left|T_{q}\right|=1 \geq 1+\sum_{T_{r} \in\left(\mathcal{N} / C_{u}^{\prime}\right)}\left\lfloor\frac{\left|T_{r}\right|}{2}\right\rfloor>2 .
$$

Therefore, it follows that, when $\left|T_{q}\right|=\left|T_{z}\right|=1$ and $C_{v}$ and $\mathcal{N} / C_{v}$ are two nonempty sets, $v$ cannot be an absorbing state.

ii. Let us consider the cases $\left|T_{q}\right|=\left|T_{z}\right| \geq 3$. As $p_{v u}=0$ implies (.13), if $\mathcal{N} / C_{w} \neq \emptyset$ then state $v$ is not absorbing, since condition (.13) does not hold. Similarly, as $p_{v w}=0$ implies (.16), if $C_{u} \neq \emptyset$ then, again, state $v$ is not absorbing since (.16) does not hold.

(b) Finally, let us consider the interesting case in which $C_{u}$ and $\mathcal{N} / C_{w}$ are two empty sets. It is clear that conditions (.13) and (.16) are trivially satisfied. Moreover, $\mathcal{N}=\left\{T_{q}, T_{z}\right\}$ and $u=0$, $v=\left|T_{q}\right|=\left|T_{z}\right|$ and $w=\left|T_{q}\right|+\left|T_{z}\right|=N$ are the only feasible states. This implies that $p_{v, u}=0$ and $p_{v, w}=0$ become sufficient conditions to prove that $v$ is an absorbing state. Since the two conditions are satisfied, we can conclude that the Markov chain $\left(M_{\mathbf{n}}, F_{\mathbf{n}}\right)$ has a third absorbing state when $\mathcal{N}=\left\{T_{q}, T_{z}\right\}$ with $T_{q}$ and $T_{z}$ two odd size tables and two absorbing states 0 and $N$ in all the other cases.

We showed that $\left(M_{\mathbf{n}}, F_{\mathbf{n}}\right)$ has an inner minimal closed set when the social space consists of two same odd-sized tables. In this case the inner minimal closed set is an absorbing state, and all the other feasible states, i.e. 0 and $N$, are also absorbing states; here the minimal closed sets consist of one single state.

In all the other cases, we have proved that no inner absorbing states can exist. It is possible to prove that minimal closed sets which are not absorbing states cannot exist either. In fact, their presence would 
require both conditions to have an inner absorbing state as those discussed above and more stringent ones. As it is well known, in Markov chains all states not belonging to minimal closed sets are transient, see, e.g. [2], and we can conclude that $\left(M_{\mathbf{n}}, F_{\mathbf{n}}\right)$ is an absorbing Markov chain.

\section{References}

[1] D. Acemoğlu, G. Como, F. Fagnani, and A. Ozdaglar. Opinion fluctuations and disagreement in social networks. Mathematics of Operations Research, 38(1):1-27, 2013.

[2] E. Behrends. Introduction to Markov chains: with special emphasis on rapid mixing. Free University of Berlin, Vieweg Verlag, Advanced Lecture Notes in Mathematics edition, 2000.

[3] L. R. Berger. A necessary and sufficient condition for reaching a consensus using DeGroot's method. Journal of the American Statistical Association, 76(374):415-418, 1981.

[4] G. I. Bischi and U. Merlone. Global dynamics in binary choice models with social influence. The Journal of Mathematical Sociology, 33(4):277-302, 2009.

[5] G. I. Bischi and U. Merlone. Binary choices in small and large groups: A unified model. Physica A: Statistical Mechanics and its Applications, 389(4):843-853, 2010.

[6] G. I. Bischi and U. Merlone. Global dynamics in adaptive models of collective choice with social influence. In G. Naldi, L. Pareschi, and G. Toscani, editors, Mathematical Modeling of Collective Behavior in Socio-Economic and Life Sciences, pages 223-244. Birkhauser, New York, NY, 2010.

[7] B. Buechel, T. Hellmann, and S. Klößner. Opinion dynamics under conformity. Working Paper, Institute of Mathematical Economics, Bielefeld University, 2013.

[8] B. Buechel, T. Hellmann, and M. Pichler. The dynamics of continuous cultural traits in social networks. Working Paper, Institute of Mathematical Economics, Bielefeld University, 2012.

[9] M. H. DeGroot. Reaching a consensus. Journal of the American Statistical Association, 69(345):118-121, 1974.

[10] M. Förster, M. Grabisch, and A. Rusinowska. Anonymous social influence. Games and Economic Behavior, 82:621-635, 2013.

[11] S. Galam. Social paradoxes of majority rule voting and renormalization group. Journal of Statistical Physics, 61(3-4):943-951, 1990. 
[12] S. Galam. Modelling rumors: the no plane Pentagon French hoax case. Physica A: Statistical Mechanics and Its Applications, 320:571-580, 2003.

[13] S. Galam. Sociophysics, A Physicist's Modeling of Psycho-political Phenomena. Understanding Complex Systems. Springer, 2012.

[14] S. Galam, Y. Gefen, and Y. Shapir. Sociophysics: A new approach of sociological collective behaviour. 1. Mean-behaviour description of a strike. The Journal of Mathematical Sociology, 9(1):1-13, 1982.

[15] O. C. Ibe. Markov processes for stochastic modeling. Elsevier Academic Press, London, UK, 2009.

[16] J. Lorenz. A stabilization theorem for dynamics of continuous opinions. Physica A: Statistical Mechanics and its Applications, 335(1):217-223, 2005.

[17] S. Moscovici, E. Lage, and M. Naffrechoux. Influence of a consistent minority on the responses of a majority in a color perception task. Sociometry, 32(4):365-380, Dec. 1969.

[18] P. Nyczka and K. Sznajd-Weron. Anticonformity or independence?-Insights from statistical physics. Journal of Statistical Physics, 151(1-2):174-202, 2013.

[19] T. C. Schelling. Hockey helmets, concealed weapons, and daylight saving: A study of binary choices with externalities. The Journal of Conflict Resolution, 17(3):381-428, 1973.

[20] D. Stauffer. A biased review of sociophysics. Journal of Statistical Physics, 151(1-2):9-20, 2012. 